\title{
Síndrome metabólico en adolescentes con sobrepeso y obesidad
}

\author{
Jaime Pajuelo ${ }^{1,2}$, Ivonne Bernui ${ }^{3}$, Violeta Nolberto ${ }^{4}$, Alberto Peña ${ }^{5}$, Luis Zevillanos ${ }^{5}$
}

Resumen

Palabras clave

\begin{abstract}
Objetivo: Conocerycuantificar la presencia de síndromemetabólicoen una población adolescente que presenta sobrepesoy obesidad. Diseño: Estudio clínico. Lugar: Servicio de Endocrinología. Hospital Nacional Dos de Mayo. Participantes: Adolescentes. Intervenciones: Se estudió una muestra de 195 adolescentes comprendidos entre los 10 a 19 años. El criterio de inclusión fue que presentasen sobrepeso u obesidad, de acuerdo al índice de masa corporal (IMC). Para ello, se hizo uso de la población de referencia de Musty col, con los siguientes criterios diagnósticos: de 85 a 95 percentil, sobrepeso; y mayor del 95 percentil, obesos. El peso, la tallay la circunferencia de la cintura (CC) fueron tomados siguiendo las recomendaciones internacionales. El síndrome metabólico fue definido mediante la recomendación de Cook(triglicéridos $>110$, colesterol HDL $<40 \mathrm{mg} / \mathrm{dL}$, glicemia $>110 \mathrm{mg} / \mathrm{dL}$ y CC > 90 percentil), la presencia de tres o más de estos criterios indicó síndrome metabólico. Principales medidas de resultados: Síndrome metabólico, sobrepeso, obesidad. Resultados: En 8,8\% de la muestra estudiada existió la presencia de síndrome metabólico. Los obesos presentaron mayor prevalencia de síndrome metabólico que los con sobrepeso (22,9 y 3\%, respectivamente); el género masculino $(24,1 \%)$ más que el femenino (2,9\%). Los de 15 años y más tuvieron un 9,7\%, con relación a los menores, 7,4\%. Conforme se incrementaba la CC, la presencia de síndrome metabólico fue mayor. Conclusiones: El síndrome metabólico se encuentra presente en la población adolescente, si bien con prevalencias bajas, pero que están íntimamente relacionadas al estado nutricional (sobrepeso y obesidad). La tendencia de estas alteraciones nutricionales es a incrementarse y como consecuencia mucho más adolescentes presentaran el síndrome metabólico, lo que lleva implícito un riesgo para su salud.
\end{abstract}

Adolescencia; obesidad; aumento de peso; metabolismo.

\begin{abstract}
Metabolic syndrome in overweight and obese adolescents

Abstract

Objective: To determine the presence of metabolic syndrome in overweight and obese adolescent population. Design: Clinical study. Setting: Endocrinology service. Hospital Nacional Dos de Mayo. Participants: Adolescents. Interventions: We studied 195 adolescents between 10 to 19 year-old. Inclusion criteria was that they should be overweight or obese. We used the reference population of Must et al. with the following diagnosis criteria: 85 to 95 percentile, overweight; higher than 95 percentile, obese.
\end{abstract}

Servicio de Endocrinología. Hospital Nacional Dos de Mayo. Lima, Perú.

Instituto de Investigaciones Clínicas. Facultad de Medicina, UNMSM. Lima, Perú.

3 Escuela Académico Profesional de Nutrición. Facultad de Medicina, UNMSM. Lima, Perú.

4 Facultad de Matemáticas, UNMSM. Lima, Perú.

5 Departamento Médico, Universidad de Piura. Piura, Perú.
Weight, height and waist circumference (WC) were taken following international recommendations. Metabolic syndrome determination followed Cook recommendations (TG $>110$, cholesterol HDL $<40 \mathrm{mg} / \mathrm{dL}$, glycemia $>110 \mathrm{mg} / \mathrm{dL}$ y $W C>90$ percentile), the presence of three or more of these criteria indicated metabolic syndrome. Main outcome measures: Setabolic syndrome, overweight, obesity. Results: Metabolic syndrome was present in $8,8 \%$ of the studied population. Those with obesity presented a higher prevalence of metabolic syndrome than those overweight (22,9 and 3\%, respectively). It occurred more in the male gender $(24,1 \%)$ than in the female $(2,9 \%)$ and in those 15 year-old and over, 9,7\% compared with the other younger group, 7,4\%. As WC increased, metabolic syndrome also increased. Conclusions: Metabolic syndrome is present in the adolescent population, although with low prevalence, but intimately related to nutritional status (overweight and obesity). Tendency of these nutritional alterations is to increase and as a consequence more and more adolescents will have metabolic syndrome that implies health risk.

Key words: Adolescence; obesity; weight gain; metabolism. 


\section{INTRODUCCIÓN}

Al síndrome metabólico ( $\mathrm{SM}$ ) se le conoce como un conjunto de factores de riesgo que son de alguna manera considerados como precursores de enfermedades relacionadas a problemas cardiovasculares y metabólicos.

Las causas del síndrome metabólico están dadas fundamentalmente por la obesidad y la resistencia a la insulina (RI) Esta es una hipótesis sustentada por una serie de estudios prospectivos, dentro de ellos se tiene The Cardiovascular Risk in Young Finns Study, que fue uno de los primeros grupos en estudiar los factores que predicen el síndrome metabólico durante la infancia. Para ello, siguieron durante 6 años a un grupo de 1865 niños y adolescentes. Los resultados mostraron que la insulinemia fue mayor en aquellos que posteriormente desarrollaron el SM, sugiriendo la idea que la insulinorresistencia precede a la aparición del mismo $\left.{ }^{(}\right)$.

Recientemente, se intentó esclarecer la posible contribución de la obesidad y la resistencia a la insulina, en el riesgo a desarrollarlo durante la edad adulta. Para ello se siguió a 718 niños, entre 8 a 17 años, durante 11 años. Aquellos que presentaban un índice de masa corporal (IMC) o una hiperinsulinemia por encima del $75 \mathrm{p}$, tenían una posibilidad 11,7 y 3,6 veces mayor, respectivamente, a presentarlo $\left({ }^{2}\right)$.

La Organización Mundial de la Salud ha calificado a la obesidad como la epidemia del siglo XXI $\left({ }^{3}\right)$. En los EE UU, en los últimos 25 años, la prevalencia se ha incrementado, de 4 a $15,3 \%$, en los niños de 6 a 11 años, y de 6 a 15,5\%, en los de 12 a 19 años $\left({ }^{3}\right)$.

En el Perú, el sobrepeso y la obesidad, en el grupo de adolescentes, también ha aumentado en estos últimos 30 años. En el género femenino, ha pasado de 11,2 a
$18,6 \%$, mientras que en el masculino pasó de 4 a $9,7 \%\left({ }^{4-5}\right)$. En lo que respecta a la resistencia a la insulina, se ha comunicado en el mismo grupo, para los que presentan sobrepeso $12,3 \%$ y en los que tienen obesidad un incremento a $16,2 \%\left({ }^{6}\right)$ Un estudio ha mostrado que, a partir de los 10 años, todo niño que presenta sobrepeso u obesidad ya tiene valores de circunferencia de la cintura por encima del 90 percentil $\left({ }^{7}\right)$ Así mismo, se ha encontrado, en niños obesos de 6 a 17 años, una elevada prevalencia de dislipidemias $\left({ }^{8}\right)$ Estos hallazgos permiten apreciar la presencia de factores de riesgo a muy temprana edad.

Sobre la base de esta información, es factible deducir que la presencia del síndrome metabólico deber ser alta y a la vez preocupante. El objetivo del presente estudio fue determinar la prevalencia del síndrome metabólico en un grupo de adolescentes con sobrepeso y obesidad.

\section{MATERIALES Y MÉTODOS}

Se estudió 195 niños adolescentes, todos ellos comprendidos entre los 10 y 19 años de edad. El criterio de inclusión fue que tuviesen sobrepeso u obesidad.

A todos se les tomó las siguientes medidas antropométricas: peso, talla y circunferencia de la cintura (CC). Con las dos primeras, se calculó el índice de masa corporal, expresado en kilogramos sobre talla cuadrado $\left(\mathrm{kg} / \mathrm{m}^{2}\right)$. El peso y la talla fueron tomadas siguiendo las indicaciones metodológicas aceptadas internacionalmente $\left({ }^{9}\right)$. La CC se hizo con las recomendaciones de la Organización Mundial de la Salud $\left({ }^{10}\right)$.

Con la finalidad de identificar el sobrepeso y la obesidad, se utilizó la tabla percentilar de Must y col $\left(^{11}\right)$ la cual relaciona la edad y el género, con los siguientes criterios diagnósticos: valores entre 85 
y 95 para el sobrepeso y mayor del percentil 95, para la obesidad.

Se dosó el perfil coronario (C-HDL y triglicéridos) y la glicemia de sangre venosa con la metodología conocida. El síndrome metabólico fue definido mediante la presencia de tres o más criterios, sobre la base de los valores dados por Cook $\left({ }^{12}\right)$, y que son los siguientes:

- Triglicéridos $>110 \mathrm{mg} / \mathrm{dL}$

- Colesterol-HDL $<40 \mathrm{mg} / \mathrm{dL}$

- Circunferencia de cintura $>90$ percentil

- Glicemia > $110 \mathrm{mg} / \mathrm{dL}$

En este trabajo no se tomó la presión arterial.

\section{RESULTADOS}

La Tabla 1 muestra las características de las poblaciones estudiadas, sobrepeso y obesidad. Se las presenta de acuerdo a medidas de tendencia central, como la media aritmética y el desvío estándar (DE). El peso, índice de masa corporal (IMC), circunferencia de la cintura (CC) y triglicéridos

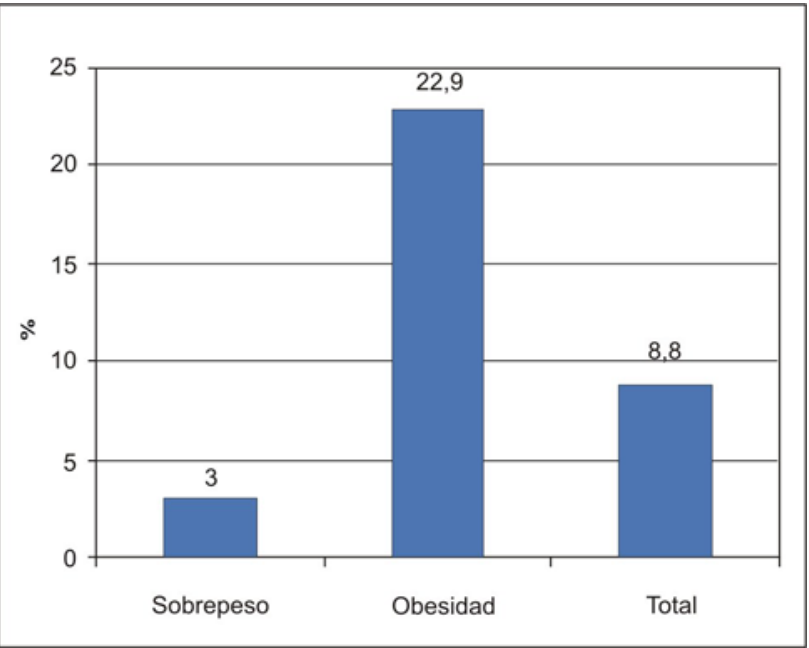

Figura 1. Prevalencia de síndrome metabólico en adolescentes, de acuerdo a estado nutricional.
Tabla 1. Promedio y desvío estándar de las variables estudiadas, de acuerdo al estado nutricional.

\begin{tabular}{cccc}
\hline & Sobrepeso & Obesidad & $\begin{array}{c}\text { Significación } \\
\text { estadística }\end{array}$ \\
\hline $\mathrm{n}$ & 135 & 57 & \\
Edad (años) & $15,1(1,7)$ & $14,2(2,6)$ & $\mathrm{NS}$ \\
Peso $(\mathrm{kg})$ & $62,7(8,4)$ & $72,8(18,5)$ & $<0,001$ \\
Talla $(\mathrm{m})$ & $1,56(0,07)$ & $1,53(0,12)$ & $\mathrm{NS}$ \\
$\mathrm{IMC}\left(\mathrm{kg} / \mathrm{m}^{2}\right)$ & $25,7(1,6)$ & $30,5(4)$ & $<0,001$ \\
$\mathrm{CC}(\mathrm{cm})$ & $81,5(6,5)$ & $92,7(12)$ & $<0,001$ \\
Tg(mg/dL) & $117,9(40,7)$ & $143(60)$ & $<0,001$ \\
CT-HDL (mg/dL) & $39,2(8,8)$ & $37,7(7)$ & NS \\
G (mg/dL) & $85,6(11,3)$ & $82,6(9,9)$ & NS \\
\hline
\end{tabular}

$(\mathrm{Tg})$ son las únicas que presentan un nivel de significancia estadística $(p<0,001)$.

En la Figura 1 se muestra la prevalencia de síndrome metabólico encontrada para las poblaciones del estudio. En la muestra en general existe $8,8 \%$ de adolescentes con síndrome metabólico, siendo más prevalente en los obesos $(22,9 \%)$ que en los con sobrepeso $(3 \%)$.

En la Figura 2 se puede observar la presencia del síndrome metabólico, de acuer-

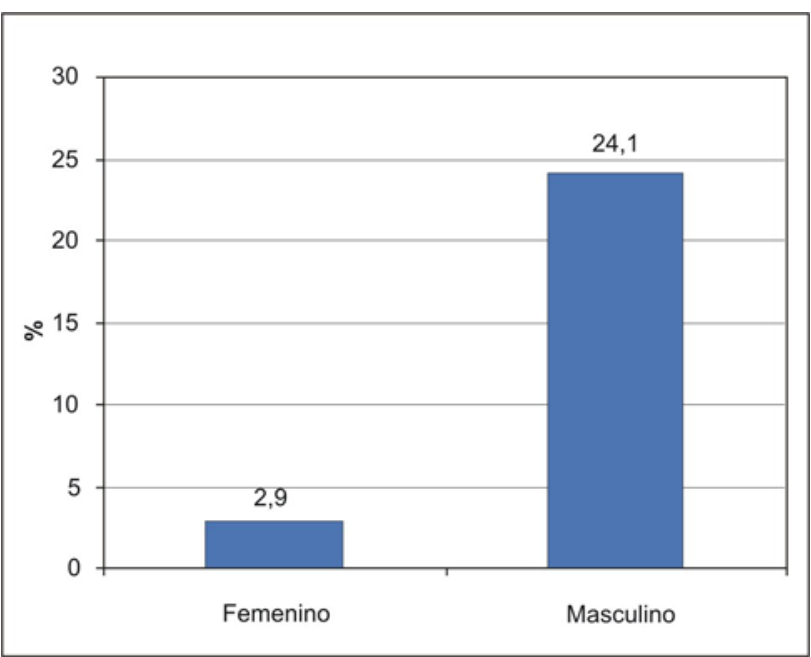

Figura 2. Prevalencia de síndrome metabólico en adolescentes, de acuerdo a género. 


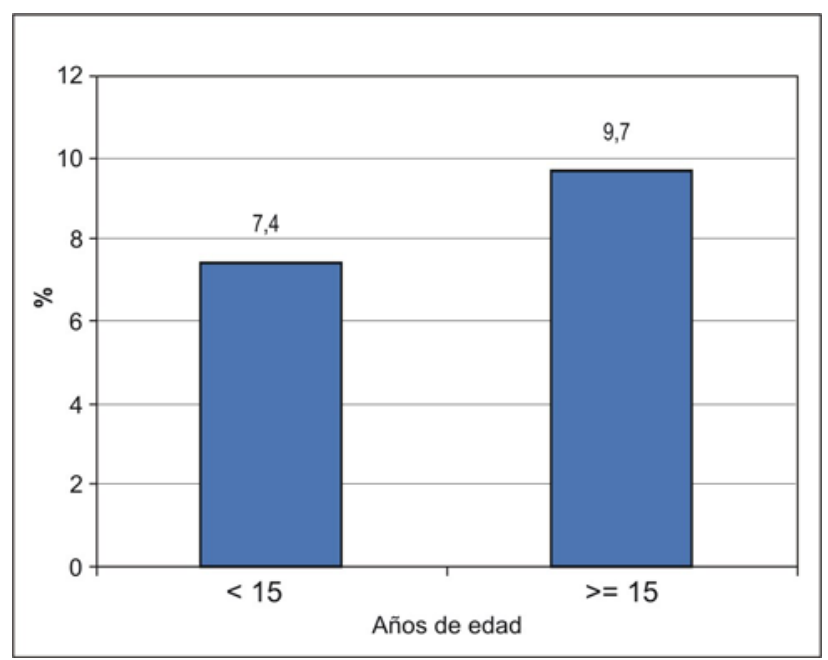

Figura 3. Prevalencia de síndrome metabólico en adolescentes, según edad.

do al género. En el género masculino se observa mayor presencia de síndrome metabólico $(24,1 \%)$ que en el femenino $(2,9 \%)$.

La Figura 3 permite observar el comportamiento del síndrome metabólico de acuerdo a grupos de edad. Ahí se muestra que en

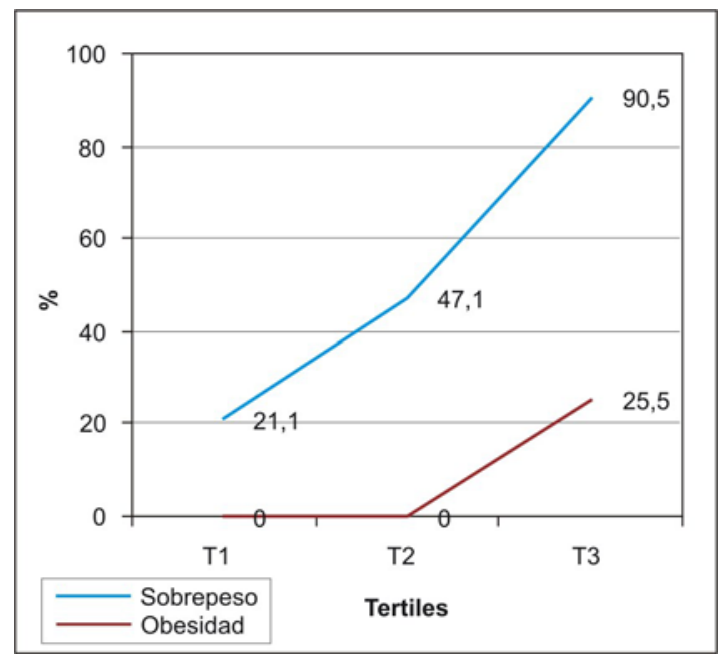

Figura 4. Prevalencia de síndrome metabólico en adolescentes, según tertiles $(\mathrm{T})$ de circunferencia de cintura. el grupo de mayor edad, en este caso los de 15 años y más, la presencia del síndrome es $9,7 \%$, en comparación a los del grupo menor, $7,4 \%$.

Desagregando a toda la población en tertiles de acuerdo a los valores de CC, se observa nítidamente que conforme la CC aumenta, la presencia de síndrome metabólico lo hace en la misma dirección y que esto se hace mucho más evidente en los obesos. (Figura 4).

\section{DISCUSIÓN}

Si bien el panorama para identificar a las personas adultas con síndrome metabólico es materia de discusión, en los niños y adolescentes lo es mucho más. En la población infantil no existe un criterio definido para el diagnóstico del síndrome metabólico. Sin embargo, una gran mayoría aplica una versión modificada de la definición del ATP III $\left({ }^{12}\right)$.

El síndrome metabólico es la expresión de una serie de anormalidades que, vistas en forma individual, acarrean un riesgo para la salud, pero que en su conjunto se potencian. El hecho dramático es que esta situación se está presentando en adolescentes, cuya exposición a estos riesgos hace pronosticar que los problemas cardiovasculares se presentarán cuando sean adultos jóvenes.

Si bien la prevalencia del síndrome metabólico encontrada en este estudio es preocupante, lo es más tomando en cuenta que, al no haberse podido obtener datos de la presión arterial, la real prevalencia pudiera ser ligeramente mayor. A pesar de esto, lo trascendente es que la tendencia del sobrepeso y la obesidad es a incrementarse y es fácil deducir que el síndrome metabólico seguirá la misma dirección.

La literatura muestra una serie de estudios que dan cuenta la presencia del sín- 
drome metabólico en este grupo poblacional. En población española de 8 a 14 años, se encontró 29,9\% ( $\left.{ }^{13}\right)$, en adolescentes del estado de Querétaro-México, fue $21,1 \%\left({ }^{14}\right)$, en la población infantil europea, la prevalencia fue $8,9 \%\left({ }^{15}\right)$, en 4811 niños de 6 a 18 años, se comunicó $14,1 \%$, encontrándose una asociación entre la actividad física y el síndrome metabólico, donde los varones son más activos $\left({ }^{16}\right)$; y, en Turquía, la prevalencia es muy baja, 2,2\% $\left({ }^{17}\right)$. Como se puede apreciar, las prevalencias son diferentes comparándolas con nuestros hallazgos.

La tendencia epidemiológica, determina que el síndrome metabólico se presenta en mayor medida en las personas que tienen mayor IMC y mayor edad. En el Perú, esta tendencia se ha encontrado en un estudio en el ámbito nacional realizado en adultos $\left({ }^{18}\right)$.

Otros estudios ya apuntan a relacionar el síndrome metabólico con el estado nutricional de los niños e independientemente de la magnitud del problema todos concuerdan en que el incremento se hace en relación directa a su IMC; o sea que, quienes más presentan el problema son los obesos, luego les siguen los que tienen sobrepeso y por último los normales, donde prácticamente no existe.

En ese sentido, Weiss informó un 38,7\% en obesidad moderada y $49,7 \%$ en obesidad severa $\left({ }^{19}\right)$. Lo mismo acontece con niños chilenos, donde se comunicó $4,3 \%$ en los con sobrepeso y $29,8 \%$ en los con obesidad $\left({ }^{20}\right)$. Un estudio en México encontró que la presencia del síndrome metabólico es más prevalente en adolescentes que tienen un mayor índice de masa corporal, $18,4 \%$ en los que tenían sobrepeso y $58,6 \%$ en los considerados obesos $\left({ }^{21}\right)$. En niños argentinos, se encontró $11,3 \%$ de síndrome metabólico, destacándose $21,9 \%$ en los niños que presentaban obesidad (22) El Third National Health and Nutrition Examination
Survey mostró la relación que existe entre el IMC y la presencia del síndrome metabólico. En adolescentes considerados como normales, de acuerdo a su IMC, se encontró $0,1 \%$; en aquellos con sobrepeso, $6,8 \%$, y en los obesos, $28,7 \%\left({ }^{12}\right)$.

Esta misma tendencia también se encuentra en países asiáticos, como por ejemplo en la India, donde se halló $4,5 \%$ de síndrome metabólico, siendo más prevalerte en aquellos con sobrepeso $(11,5 \%)$ y en los obesos $(36,6 \%){ }^{(23)}$. Lo mismo sucedió en adolescentes iraníes, donde informaron $10,1 \%$ de síndrome metabólico, destacándose su presencia en relación directa al IMC, en normales 4,8, sobrepeso 20,1 y obesos $56,6 \%$, de acuerdo a los puntos de corte de Cole $\left({ }^{24}\right)$.

Todos estos estudios muestran el peligro que acarrea el aumento de peso. Sus resultados son concluyentes y concuerdan plenamente con los encontrados en el presente estudio. Si bien el IMC no es considerado como criterio diagnóstico para identificar síndrome metabólico, se lo debe tomar muy en cuenta dentro de la categoría de riesgo.

Se calcula que entre 50 y $80 \%$ de adolescentes obesos continúan siendo obesos en su edad adulta. La razón de probabilidad de ser obeso en la adultez aumenta progresivamente a medida que el IMC se incrementa en los adolescentes. Las personas de un IMC muy alto durante la adolescencia mostraron un riesgo de morir al llegar a la adultez de 30 a $40 \%$ mayor que las personas con un IMC moderado $\left({ }^{25}\right)$

La información comunicada sugiere que los diagnósticos de síndrome metabólico deben ser lo más temprano posible, con el objeto de poder realizar intervenciones oportunas que permitan evitar la aparición de enfermedades cardiovasculares.

En el presente estudio, los criterios de mayor presencia para identificar el síndro- 
me metabólico fueron el incremento de la CC y las alteraciones en el colesterol HDL. Un hecho similar fue encontrado en un estudio llevado a cabo en Los Ángeles, en adolescentes descendientes de hispanos, donde se halló una prevalencia de síndrome metabólico de $30 \%\left({ }^{26}\right)$.

Otra situación a tener muy en cuenta son los valores de CC, no solo por ser uno de los criterios para diagnosticar síndrome metabólico, sino por la repercusión que tiene como indicador de riesgo cardiovascular. En ese sentido, el incremento de la CC en los niños ha mostrado un correlato con niveles elevados de colesterol total, de triglicéridos, de insulina, como también bajas concentraciones de colesterol HDL $\left({ }^{27,28}\right)$ Por otro lado, lo encontrado muestra que quienes presentan valores altos de CC (tercer tertil) tienen mayor presencia de síndrome metabólico que en los anteriores tertiles.

La CC es el mejor indicador antropométrico de carácter predictivo de enfermedades cardiovasculares. Por esa razón, se lo considera como uno de los criterios más importantes para definir síndrome metabólico. En ese sentido, se puede inferir que los incrementos que se dan en función del tiempo van a repercutir en un aumento del síndrome metabólico. La tendencia que se está observando en muchas partes del mundo, por ejemplo, en niños de EE UU $\left({ }^{29}\right)$, de Australia $\left({ }^{30}\right)$, Reino Unido $\left.{ }^{(31}\right)$ y España $\left({ }^{32}\right)$, así lo determinan.

Sin lugar a dudas, e independientemente de no ser considerado como criterio diagnóstico para el síndrome metabólico, el sobrepeso y la obesidad es lo primero que hay que enfrentar. Dos son las medidas conocidas ampliamente para hacerlo, mejorar los patrones dietarios y promover la actividad física, esto último a su vez permitiría mejorar los valores del colesterol HDL, que es el otro componente de mayor presencia en el diagnóstico del SM.

\section{REFERENCIAS BIBLIOGRÁFICAS}

1. Mills G, Avery P, McCarthy M, Hattersley A, Levy J, Timan G. Heritability estimates for beta cell function and features of the insulin resistance syndrome in UK families with an increased susceptibility totypr 2 diabetes. Diabetologia. 2004;47:732-8.

2. Srinivisan S, Myers I, Berenson G. Predictability of childhood adiposity and insulin for developing insulin resistance syndromes in young adulthood. The Bogalusa Heart Study. Diabetes. 2002;51:204-9.

3. Ogden C, Flegal K, Carrol M, Johnson C. Prevalence and trends in overweight among US children and adolescents. JAMA. 2002;288:1728-32.

4. Pajuelo J. La obesidad infantil en el Perú. Lima: Facultad de Medicina-UNMSM; 2003.

5. Pajuelo J, Acevedo M. La situación nutricional de niños de 8 a 14 años en el Perú. Consensus. 2006;11:9-16.

6. Pajuelo J, Pando R, Leyva M, Hernandez K, Infantes R. Resistencia a la insulina en adolescentes con sobrepeso y obesidad. An Fac Med. 2006;67:23-9.

7. Pajuelo J, Canchari E, Carrera J, Leguia D. La circunferencia de la cintura en niños con sobrepeso y obesidad. An Fac Med. 2006;65:167-71.

8. Pajuelo J, Rocca J, Gamarra M. Obesidad Infantil: Características antropométricas y bioquímicas. An Fac Med. 2003;64(1):21-6.

9. Lohman T, Roche A. Anthropometric Standardization Reference Manual. Illinois: Human Kinects Books; 1990.

10. Wang J, Thornton JC, Bari S, Williamson B, Gallagher D, Heymsfield SB, et al. Comparisons of waist circumferences measured at 4 sites. Am J Clin Nutr. 2003;77:379-84.

11. Must A, Dallal G, Dietz W. Reference data for obesity: $85 \mathrm{p}$ and $95 \mathrm{p}$ percentiles of body mass index (wt/ht2)-a correction. Am J Clin Nutr. 1991;54:773.

12. Cook S, Weitzman M, Auinger P, Nguyen M, Dietz W. Prevalence of a metabolic syndrome phenotype in adolescents. Arch Pediatr Adolesc Med. 2003;157:821-7.

13. Bueno G, Bueno O, Moreno L, Garcia R, Trajaco B, Garagorri J, Bueno M. Diversity of metabolic síndrome risk factors in obese children and adolescents. J Physiol Biochem. 2006;62(2):125-33.

14. Aguilera A. Síndrome Metabólico en adolescentes en el Estado de Querétaro-México. Simposio 2006. Revista Salud Pública y Nutrición [revista en Internet]. 2006;(17) [acceso 13 marzo de 2007]. Disponible en: http://www.respyn.uanl.mx/ especiales/2006/ee-17-2006/index.html

15. Csabi G, Torok K, Jeges S, Melnar D. Presence of metabolic cardiovascular syndrome in obese children. Eu J Pediatric. 2000;159:91-4.

16. Kelishadi R, Razaghi EM, Gouya MM, Ardalan G, Gheiratmand R, Delavari A, et al. Association of physical activity and the metabolic syndrome in children and adolescents: CASPIAN Study. Horm Res. 2007;67(1):46-52. 
17. Agisbasli M, Cakir S, Ozme S, Ciliv G. Metabolics syndrome in Turkish children and adolescents. Metabolism. 2006;55(8):1002-6.

18. Pajuelo J, Sanchez J. El síndrome metabólico en adultos, en el Perú. An Fac Med. 2007;68:38-46.

19. Weiss R, Dziura J, Burgest T. Obesity and the metabolic syndrome in the children and adolescent. NJEM. 2004;350:2362-70.

20. Burrrow R, Leiva L, Wiestaub G, Ceballos X, Gattes V, Lora L, Albela C. Síndrome metabólico: asociación con sensibilidad insulínica y con magnitud y distribución de la obesidad. Revista Médica de Chile. 2007;135:174-81.

21. Posadas C. Obesidad y el síndrome metabólico en niños y adolescentes. Revista de Endocrinología y Nutrición. 2005;13:45-6.

22. Hirschler V, Calcagno M, Aranda S, Maccallini G, Jadzinsky M. Síndrome Metabólico en la infancia y su asociación con resistencia a la insulina. Arch Argent Pediatr. 2006;104:48691.

23. Singh R, Bhansali A, Sialey R, Aggarwal A. Prevalence of metabolic syndrome in adolescents from a north Indian population. Diabet Med. 2007;24:195-9.

24. Esmaillzadeh A, Mismizan P, Azadbakit L, Etemadi A, Azizi F. High prevalence of the metabolic syndrome in Iranian adolescents. Obesity. 2006;14:377-82.

25. Cruz M, Weigensberg M, Huang T, Ball G, Shaibi G, Goran M. The metabolic syndrome in overweight Hispanic youth and the role of insulin sensitivity. J Clin Endocrinol Metab. 2004;89(1):108-13.

26. Engeland A Bjorge T, Tverdar A, Segard A. Obesity in adolescence and adulthood and the risk of adult mortality. Epidemiology. 2004;15(19):79-85.

27. Freedman D, Serdula M, Srinivasan S, Berenson G. Relation of circunferences and skinfold thickness to lipid and insulin concentrations in children and adolescents: the Bogalusa Herat Study. Am J Clin Nutr. 1999;69:308-17.

28. Savva S, Tornaritis M, Savva M. Waist circumference and waist-to-height ratio are better predictors of cardiovascular disease risk factor in children than body mass index. Int $\mathrm{J}$ Obes Relat Metab Disord. 2000;24:1453-8.

29. Chaoyang L, Ford E, Mokdad H, Cook S. Recent trends in waist circumference and waist-height ratio among US children and adolescents. Pediatrics. 2006;118:1390-8.

30. Garnett S, Coweil C, Baur L. Increasing central adiposity: the Neppan longitudinal study of young people aged 7-8 to 12-13 y. Int J Obes. 2005;29:1353-60.

31. McCarthy H, Jarrett K, Emmett P, Regan I. Trends in waist circumference in young British children a comparative study. Int J Obes. 2005;29:157-62.

32. Moreno L, Sarria A, Fleta J, Marcos A, Bueno M. Secular trends in waist circunference in spanish adolescents 1995 to 2002. Arch Dis Child. 2005;90:818-9.

Manuscrito recibido el 17 de julio de 2007 y aceptado para publicación el 04 de setiembre de 2007.

Correspondencia:

Jaime Pajuelo Ramírez.

Instituto de Investigaciones Clínicas

Hospital Nacional Dos de Mayo.

Av. Grau cuadra 13. Parque Historia de la Medicina.

Lima 1, Perú.

Correo-e: saturse@speedy.com.pe 